Katherine N. Weller, MD

Department of Allergy and Clinical Immunology,

Respiratory Institute, Cleveland Clinic,

Cleveland, $\mathrm{OH}$
Fred H. Hsieh, MD

Department of Allergy and Clinical Immunology,

Respiratory Institute, Cleveland Clinic,

Cleveland, $\mathrm{OH}$

\title{
Anaphylaxis: Highlights from the practice parameter update
}

\section{ABSTRACT}

The practice parameter update on anaphylaxis from the Joint Task Force on Practice Parameters, with the collaboration of the American Academy of Allergy, Asthma, and Immunology and the American College of Allergy, Asthma, and Immunology, addresses key issues in the management and prevention of anaphylaxis. The updated guidelines define diagnostic criteria for anaphylaxis; therapeutic use of epinephrine, antihistamines, and glucocorticoids; prevention of recurrent anaphylaxis; and follow-up care that includes education on trigger avoidance and use of self-injectable epinephrine.

\section{KEY POINTS}

Epinephrine is first-line pharmacotherapy for anaphylaxis.

Epinephrine should be administered at the onset of anaphylaxis as delays often increase risks for morbidity and mortality.

Patients with severe anaphylaxis should be observed for biphasic anaphylaxis, even after signs and symptoms resolve.

The updated guidelines are expected to improve outcomes by emphasizing early treatment with epinephrine and identifying risk factors for severe and biphasic anaphylaxis.
T HE UPDATED GUIDELINES on the prevention and treatment of anaphylaxis ${ }^{1}$ from the Joint Task Force on Practice Parameters address key issues in the prevention and management of anaphylaxis, including diagnostic criteria for anaphylaxis; therapeutic use of epinephrine, antihistamines, and glucocorticoids; prevention of recurrent anaphylaxis; and follow-up care including patient education on trigger avoidance and use of self-injectable epinephrine. This update to the 2015 guidelines $^{2}$ was a collaborative effort of the American Academy of Allergy, Asthma, and Immunology and the American College of Allergy, Asthma, and Immunology.

\section{GENERAL CONSIDERATIONS OF THE UPDATE}

Anaphylaxis is an acute, potentially life-threatening allergic emergency that can present with diverse symptoms that often but not always involve hemodynamic compromise. The updated guidelines note that anaphylaxis is highly likely in a patient experiencing at least 1 of the following 3 clinical scenarios (Table 1$)^{1}$ :

- Acute onset of symptoms involving the skin or the mucous membrane, or both, including hives, itching, flushing, or swelling; plus the acute onset of respiratory symptoms, with or without hypotension or other symptoms of target-organ dysfunction

- Involvement of 2 or more organ systems that occurs rapidly after exposure to a likely allergen, including skin or mucosal membrane symptoms, respiratory symptoms, hypotension or other symptoms of target-organ dysfunction, and sudden gastrointestinal symptoms 
- Hypotension occurring acutely after exposure to a known or established allergen for that patient.

In patients who meet the anaphylaxis criteria, epinephrine is recommended. However, the updated guidelines recognize that epinephrine treatment may still be appropriate in some patients who do not meet anaphylaxis criteria, such as a patient exposed to a likely allergen who develops symptoms in a single organ system. Anaphylaxis is considered to be severe if respiratory failure or cardiovascular collapse occurs.

Risk factors for severe anaphylaxis include cardiovascular disease, asthma, older age, and additional coexisting or comorbid conditions such as atopy, concomitant beta-blocker or angiotensin-converting enzyme (ACE) inhibitor use, or an established mast cell disorder such as bonemarrow biopsy-proven systemic mastocytosis. ${ }^{1}$

In adults, the most common causes of anaphylaxis are medications and stinging insects. In children, foods and stinging insects are the most common triggers. Food allergy is present in $8 \%$ to $11 \%$ of the US population, and adverse drug reactions occur in up to $10 \%$ of the population and in up to $20 \%$ of hospitalized patients. ${ }^{1}$

\section{ANAPHYLAXIS TREATMENT}

Epinephrine is the first-line therapy for anaphylaxis, and its administration should not be delayed, as delays are associated with higher rates of morbidity and mortality. There are no absolute contraindications to epinephrine use, even in pregnant patients or those with coronary artery disease or tachyarrhythmia.

Epinephrine should be administered at the onset of anaphylaxis, intramuscularly in the mid-outer thigh at a dose of $0.01 \mathrm{mg} / \mathrm{kg}$ of a $1: 1000(1-\mathrm{mg} / \mathrm{mL})$ solution, up to a maximum dose of $0.5 \mathrm{mg}$ in adults and $0.3 \mathrm{mg}$ in children. ${ }^{1}$

The most commonly used epinephrine autoinjectors are the following:

- EpiPen $0.3 \mathrm{mg} / 0.3 \mathrm{~mL}$ for adult patients

- EpiPen Jr $0.15 \mathrm{mg} / 0.15 \mathrm{~mL}$ for pediatric patients weighing 15 to $30 \mathrm{~kg}$

- Auvi-Q $0.3 \mathrm{mg} / 0.3 \mathrm{~mL}$ for adults

- Auvi-Q $0.15 \mathrm{mg} / 0.15 \mathrm{~mL}$ for pediatric patients weighing 15 to $30 \mathrm{~kg}$

- Auvi-Q (0.1 mg/0.1 mL) for pediatric patients weighing 7.5 to $15 \mathrm{~kg}$.

If possible, the inciting allergen should be re-
TABLE 1

\section{Diagnostic criteria for anaphylaxis}

Anaphylaxis is highly likely when any 1 of the following 3 criteria is met:

1 Sudden onset of symptoms (minutes to several hours) with involvement of skin and/or mucosa (eg, generalized hives, itching or flushing, swollen lips/tongue/uvula), AND at least one of the following:

Respiratory symptoms or signs (eg, shortness of breath, wheezing, cough, hypoxemia)

Hypotension or other symptoms of target-organ dysfunction (eg, collapse, incontinence)

Sudden onset of 2 or more of the following after exposure to a likely allergen or other trigger for that patient (minutes to several hours):

Skin or mucosal membrane symptoms

Respiratory symptoms

Hypotension or other symptoms of target-organ dysfunction

Gastrointestinal symptoms such as abdominal pain or vomiting

3 Hypotension occurring acutely (minutes to several hours) after exposure to a known or established allergen for that patient. Hypotension is defined as the following:

Adults: Systolic blood pressure $<90 \mathrm{~mm} \mathrm{Hg}$, or a decrease $>30 \%$ from patient's baseline

Infants and children: Low systolic blood pressure (age-specific) or a decrease $>30 \%$ in systolic pressure

Based on information from reference 1 .

moved. Airway, breathing, and circulation should then be assessed, appropriate assistance summoned, and cardiopulmonary resuscitation begun, if needed. ${ }^{2}$ Additional interventions include placing the patient in a supine position (left side for pregnant patients), providing supplemental oxygen, and administering intravenous fluid resuscitation to patients with hypotension.

Second-line therapy with beta-2 agonists such as albuterol, antihistamines (histamine-1 and histamine-2 receptor antagonists), and corticosteroids may be considered. Glucagon may be helpful for patients who are receiving beta-adrenergic blocking agents. ${ }^{2}$ Unlike epinephrine, these second-line medications will 
not effectively treat cardiovascular symptoms such as hypotension and should not be administered in place of epinephrine. ${ }^{1}$

After treatment of anaphylaxis, monitor the patient until signs and symptoms have fully resolved. Extended observation is suggested for patients with severe anaphylaxis and those who require more than 1 dose of epinephrine. This is indicated to monitor for a potential biphasic reaction. ${ }^{1}$

Any patient who has experienced anaphylaxis should be evaluated by an allergy and immunology specialist to determine the causative agent, if any. It is not possible to predict the severity of any future event based on the severity of past events. Therefore, consider prescribing an epinephrine autoinjector to patients who have experienced anaphylaxis. Instruct patients on the use of the device, and educate them on the risk of anaphylaxis recurrence and trigger avoidance.

\section{BIPHASIC ANAPHYLAXIS}

Biphasic anaphylaxis - a clinical condition in which the symptoms of anaphylaxis recur after medical resuscitation, recovery of vital signs, and resolution of all signs and symptoms-is estimated to occur in $1 \%$ to $20 \%$ of patients and may occur up to 72 hours after resolution of anaphylaxis. ${ }^{1}$ Intramuscular epinephrine is the first-line treatment for both the initial and the delayed reaction.

The most significant risk factor for experiencing a biphasic anaphylactic reaction is a severe initial anaphylactic reaction or the need for more than 1 dose of epinephrine. Other risk factors include a wide pulse pressure at initial presentation, unknown anaphylaxis trigger, cutaneous signs and symptoms (including and urticaria and angioedema), delayed time of administration of the first epinephrine dose ( $>60$ minutes), and presence of a drug trigger in pediatric patients. ${ }^{1}$

Thus, patients presenting with severe anaphylaxis, especially those requiring more than 1 dose of epinephrine, should be considered for longer direct observation given the potential for biphasic anaphylaxis, even after complete resolution of signs and symptoms of anaphylaxis. From a clinical practice standpoint, for patients with no severe risk factors, a 1-hour asymptomatic observation period may be reasonable. For patients at higher risk, 6 hours or longer should be strongly considered.

\section{Preventing biphasic anaphylaxis}

There are no reliable interventions to prevent biphasic anaphylaxis. Antihistamines and glucocorticoids are commonly used for emergency treatment of urticaria, itching, and swelling. However, if anaphylaxis is not recognized and those medications are administered instead of epinephrine therapy, it could delay the start of firstline anaphylaxis treatment with epinephrine.

In addition, the 2020 Joint Task Force did not identify any benefit of antihistamines or glucocorticoids in preventing biphasic anaphylaxis. Rather, it found that glucocorticoid use may actually be associated with increased risk for biphasic anaphylaxis in children, though confounding with severity could not be excluded. ${ }^{1}$

\section{ADDRESSING THE POTENTIAL CAUSES OF ANAPHYLAXIS}

\section{Chemotherapy}

The incidence of anaphylaxis has increased during some chemotherapy protocols that include agents such as pegaspargase, docetaxel, carboplatin, oxaliplatin, and paclitaxel. ${ }^{1}$ Premedication with glucocorticoids or antihistamines has been shown to significantly decrease the rate of hypersensitivity reactions to chemotherapy. ${ }^{1}$ Therefore, premedication is recommended to decrease the risk of hypersensitivity reactions during these protocols, including prevention of infusion-related reactions in patients who have not previously experienced a reaction to the drug. Premedication may also be considered for some biologics, such as rituximab used to treat autoimmune disorders and B-cell malignancies (eg, diffuse large B-cell non-Hodgkin lymphoma, follicular B-cell non-Hodgkin lymphoma). Of note, this is a conditional recommendation with a very-low-certainty rating of evidence, as studies have not included patients who have experienced anaphylaxis to those drugs. ${ }^{1}$

\section{Radiocontrast media}

The current standard approach to reducing hypersensitivity reactions to radiocontrast me- 
dia in patients with a history of radiocontrast reactions involves premedication with antihistamines and glucocorticoids. The updated guidelines highlight recent studies that suggest the evidence supporting premedication to prevent hypersensitivity reactions is not definitive in patients with a history of contrast reactions who are scheduled to receive low- or iso-osmolar, nonionic radiocontrast media. ${ }^{1}$ This is based on analyses that suggest the greatest risk reduction in patients with anaphylaxis from ionic, hyperosmolar radiocontrast media may be derived from using low-osmolar, nonionic contrast agents rather than hypo-osmolar, nonionic media plus pretreatment with high-dose glucocorticoids. A meta-analysis described in the guidelines did not find a clear benefit from premedication with a histamine-1 receptor antagonist plus a glucocorticoid in these patients. However, this is a conditional recommendation with a very-low-certainty rating of evidence.

The guidelines continue to suggest consideration of a premedication regimen for patients with a high level of perceived risk of anaphylaxis or comorbidities associated with greater anaphylaxis fatality risk, such as underlying cardiovascular disease, use of beta-blockers, or history of severe anaphylaxis. ${ }^{1}$ The use of other strategies (such as rapid desensitization) to treat or prevent delayed reactions to radiocontrast media is not addressed in the current guidelines. In addition, management strategies involving use of non-cross-reactive radiocontrast media without glucocorticoid premedication are proposed, but substantive prospective trials are lacking.

\section{Subcutaneous allergen immunotherapy}

In patients receiving venom immunotherapy, ACE inhibitors have been associated with an increased frequency of reactions and should be discontinued whenever possible. Betaadrenergic blocking agents have also been associated with a higher severity of events and are thought to interfere with the efficacy of epinephrine, and their discontinuation should be considered. ${ }^{2}$ A very-low-certainty rating of evidence suggests that premedication with glucocorticoids or antihistamines does not significantly reduce the risk of a hypersensitivity reaction in patients receiving allergen immunotherapy; however, there may be some benefit to premedication in patients undergoing immunotherapy procedures with a high baseline rate of systemic reactions.

\section{WHAT'S DIFFERENT FROM THE 2015 GUIDELINES?}

The Joint Task Force used the Grading of Recommendations, Assessment, Development, and Evaluation (GRADE) analysis to rigorously evaluate the certainty of the literature to answer key questions, ${ }^{1}$ whereas the 2015 guidelines task force classified recommendations by the strength of the recommendations and the quality of the evidence. ${ }^{2}$

The updated guidelines state that anaphylaxis can present with a variety of clinical manifestations and recommend treatment with epinephrine in patients who experience symptoms after exposure to a likely allergen. They expand and clarify the definition of anaphylaxis and include recommendations for pediatric patients as well.

There was concern that the definition of anaphylaxis in previous guidelines would exclude patients who did not meet certain criteria, such as a patient exposed to a likely allergen who develops symptoms in a single organ system. The updated guidelines address this to promote early treatment of anaphylaxis for more patients.

Compared with previous guidelines, the update more directly addresses the evidence and rationale underlying the recommended approach to treatment and monitoring of patients with biphasic anaphylaxis in the rigorous GRADE format. The guidelines also address the identification and mitigation of risk factors for biphasic anaphylaxis, which was not fully elucidated in prior guidelines. ${ }^{1}$

The guidelines also update recommendations regarding premedication with antihistamines or glucocorticoids based on recent evidence that supports their role for patients undergoing specific chemotherapy protocols and rush aeroallergen immunotherapy (ie, a technique for rapidly advancing the dose of aeroallergen allergy shots to the maintenance dose over a short period of time). However, the task force did not find a convincing benefit in their use to prevent recurrent radiocontrast media anaphylaxis in patients who require lowosmolar or iso-osmolar contrast. ${ }^{1}$
Any patient who has experienced anaphylaxis should be evaluated by an allergy and immunology specialist to determine the causative agent, if any 


\section{TABLE 2}

\section{Key clinical practice recommendations from the 2020 anaphylaxis guidelines}

Treatment of anaphylaxis

Administer epinephrine as first-line pharmacotherapy for uniphasic or biphasic anaphylaxis.

Do not delay the administration of epinephrine for anaphylaxis.

All patients with anaphylaxis should receive education about anaphylaxis, risk of recurrence, trigger avoidance, self-injectable epinephrine, and thresholds for further care. Patients should also be referred to an allergist for follow-up evaluation.

\section{Biphasic anaphylaxis}

Risk factors for biphasic reactions include severe anaphylaxis or the need for more than 1 dose of epinephrine to treat anaphylaxis, wide pulse pressure, unknown anaphylaxis trigger, cutaneous signs and symptoms, and drug triggers in children.

Antihistamines and glucocorticoids are not reliable interventions to prevent biphasic anaphylaxis but may be considered as second-line treatment.

After treatment, all patients with anaphylaxis should be kept under observation until symptoms have fully resolved.

Extended observation beyond resolution of symptoms is suggested for patients who had severe anaphylaxis or required more than 1 dose of epinephrine.

Use of histamine-1 antihistamines and glucocorticoids to prevent anaphylactic reactions

Premedication with antihistamine or glucocorticoid or both in specific chemotherapy protocols and specific aeroallergen immunotherapy procedures may decrease the risk of systemic reactions.

Patients with a history of reactions to radiocontrast media should receive low- or iso-osmolar radiocontrast media with future procedures to reduce the risk of future reactions. Routine glucocorticoid or antihistamine premedication may not reduce the risk of hypersensitivity reactions, though they should be considered in patients perceived to be at high risk of anaphylaxis or with comorbidities that increase the anaphylaxis fatality risk.

Based on information from reference 1

\section{WHAT'S THE EXPECTED CLINICAL IMPACT?}

The updated guidelines will likely improve outcomes in patients with anaphylaxis by emphasizing early treatment with epinephrine and identifying risk factors for severe and biphasic anaphylaxis. Premedication with antihista- mines and glucocorticoids may be utilized less to prevent recurrent radiocontrast media anaphylaxis, especially in patients with comorbidities that increase their risk of adverse effects from these premedication agents. These patients would include those with diabetes mellitus who are at high risk for hyperglycemia from highdose glucocorticoids. On the other hand, premedication may become more standard practice for certain chemotherapy protocols.

\section{DO OTHER SOCIETIES AGREE OR DISAGREE?}

The international anaphylaxis guidelines of the World Allergy Organization provide recommendations similar to those of the American Academy of Allergy, Asthma, and Immunology and the American College of Allergy, Asthma, and Immunology, but also address global issues such as the challenge of patient access to epinephrine autoinjectors in some countries. $^{3}$

\section{HOW WILL THE GUIDELINES CHANGE DAILY PRACTICE?}

The expanded diagnostic criteria for anaphylaxis will likely lead to earlier recognition of the condition and earlier use of epinephrine. Educating patients about unusual symptoms should enable them to identify anaphylaxis earlier and get rapid treatment.

Identifying risk factors for biphasic anaphylaxis will help clinicians target the appropriate patient population for education and consideration of antihistamines or glucocorticoids as secondary treatment. See Table 2 for a summary of key clinical guideline recommendations.

\section{WHEN DO THE GUIDELINES NOT APPLY?}

The updated guidelines were published prior to the US Food and Drug Administration approval of Palforzia, a food-desensitization product for peanut anaphylaxis. ${ }^{4}$ Therefore, the guidelines do not provide up-to-date recommendations regarding food desensitization as a therapeutic option for patients at risk for food anaphylaxis to peanuts.

\section{DISCLOSURES}

The authors report no relevant financial relationships which, in the context of their contributions, could be perceived as a potential conflict of interest. 


\section{REFERENCES}

1. Shaker MS, Wallace DV, Golden DBK, et al. Anaphylaxisa 2020 practice parameter update, systematic review, and grading of recommendations, assessment, development and evaluation (GRADE) analysis. J Allergy Clin Immunol 2020; 145(4):1082-1123. doi:10.1016/j.jaci.2020.01.017

2. Lieberman P, Nicklas RA, Randolph C, et al. Anaphylaxisa practice parameter update 2015. Ann Allergy Asthma Immunol 2015; 115(5):341-384. doi:10.1016/j.anai.2015.07.019

3. Cardona V, Ansotegui IJ, Ebisawa M, et al. World allergy organization anaphylaxis guidance 2020. World Allergy Organ J 2020; 13(10):100472.

doi:10.1016/j.waojou.2020.100472

4. Peanut allergen powder (Palforzia). JAMA 2020; 324(2):192-193. doi:10.1001/jama.2020.3599

Address: Fred H. Hsieh, MD, Department of Allergy and Clinical Immunology, Respiratory Institute, A90, Cleveland Clinic, 9500 Euclid Avenue, Cleveland, OH 44195; hsiehf@ccf.org 